\title{
Swarm shape and its dynamics in a predator-swarm model
}

\author{
Hayley Tomkins* \\ Advised by Theodore Kolokolnikov ${ }^{\dagger}$
}

June 25,2015

\begin{abstract}
We consider a particle predator-swarm model introduced in [1]. In the continuum limit of many prey particles, we develop a numerical method which tracks the boundary of the swarm. We use this method to explore the variety and complexity of swarm shapes. We also consider a special limiting case where the predator is moving inside an infinite sea of prey. Two subcases are studied: one where the predator is moving along a straight line, and another where the predator is moving in a circle. We observe various topological changes in the swarm shape as the predator speed increases, such as the appearance of an infinite tail for a predator moving in a straight line when its speed is large enough.
\end{abstract}

\section{Introduction}

Recently there have been many attempts to produce swarm models that have both the accuracy and complexity to be meaningful and the simplicity to be analysed. Each model uses a slightly different approach. Some researchers focus on specific animal groupings, such as shoals of fish, [2,3,4], flocks of birds, [5], and locust swarms, [6, 7, 8, 9]. Many propose an individual-based model $[10,11,8]$. As well, others opt to build their model depending on collected data $[12,13,14]$. One approach uses force matching, which one can view as "a regression analysis where a hypothetical rule set is optimally fitted to observed data" [15], to deconstruct observed behaviours and classify specific interactions involved [15]. Another approach uses a game-like model that designates points to predators and prey based on the nature of their interactions, interpreting dynamics from the strategies that develop [16]. Researchers have also used Particle Swarm Optimization algorithms to model swarms [17, 18]. In particular, we highlight the model Silva et. al. proposed in [19]. In [19], Silva et. al introduced a single predator to their model, considering a similar situation as we explore in this paper. More in-depth overviews of the many methods and areas of study approaching this problem are found in $[20,21]$. An excellent and thorough overview of the different modelling approaches is found in [22].

The variability of these models owes partially to the diversity of fields considering the problem of accurately modelling swarm interactions; researchers most often approach this problem from biology, mathematics, physics, and engineering [22]. Despite these variations, recurring features exist between these models. The ultimate goal of these observations is generally to model changing population numbers in between a group of predators and a linked group of prey [23]. There is usually "a short-range repulsion, a longer-range attraction, and/or an alignment among interacting agents" [2].

One of the short-comings of many of these models is that they are difficult to analyse mathematically. To address this shortcoming Chen and Kolokolnikov [1] proposed a "minimal" model of prey-swarm interactions. This model exhibits many of the key aspects of predator-prey interactions while being as simple as possible. This makes the model amenable to mathematical (and not just numerical) analysis. The model is

$$
\begin{gathered}
\frac{d x_{j}}{d t}=\frac{1}{N} \sum_{k=1, k \neq j}^{N}\left(\frac{x_{j}-x_{k}}{\left|x_{j}-x_{k}\right|^{2}}-a\left(x_{j}-x_{k}\right)\right)+b \frac{x_{j}-z}{\left|x_{j}-z\right|^{2}}, \\
\frac{d z}{d t}=\frac{c}{N} \sum_{k=1}^{N} \frac{x_{k}-z}{\left|x_{k}-z\right|^{p}} .
\end{gathered}
$$

*Department of Mathematics and Statistics, Dalhousie University, Halifax, NS, Canada, B3H 4R2. Email: hayleytomkins@dal.ca.

${ }^{\dagger}$ Department of Mathematics and Statistics, Dalhousie University, Halifax, NS, Canada, B3H 4R2. Email: tkolokol@gmail.com. 
Here, $x_{j}$ are (two-dimensional) prey positions while $z$ is the position of a single predator. $N$ is the number of prey. Chen and Kolokolnikov [1] associated the parameter $a$ with prey-prey attraction, the parameter $b$ with predator-prey repulsion, the parameter $c$ with predator-prey interactions, and the parameter $p$ loosely with the predator sensitivity to prey [1]. In the paper [1], the authors characterized the steady states of the system and their stability.

The reason for the particular choice of nonlinearities in (1) becomes clear when one considers the continuum limit of large $N$, which results in the non-local integro-differential equation model [1]:

$$
\begin{aligned}
& \rho_{t}(x, t)+\nabla \cdot(\rho(x, t) v(x, t))=0 ; \quad \int_{\mathbb{R}^{2}} \rho(y, t) d y=1 \\
& v(x, t)=\int_{\mathbb{R}^{2}}\left(\frac{x-y}{|x-y|^{2}}-a(x-y)\right) \rho(y, t) d y+b \frac{x-z}{|x-z|^{2}} \\
& \frac{d z}{d t}=c \int_{\mathbb{R}^{2}} \frac{y-z}{|y-z|^{p}} \rho(y, t) d y .
\end{aligned}
$$

Here, $\rho(x, t)$ is the density of the swarm and $v(x, t)$ is its velocity field.

The divergence of the velocity then yields

$$
\begin{aligned}
\nabla \cdot v & =\int_{\mathbb{R}^{2}}(\Delta \ln |x-y|-2 a) \rho(y, t) d y+b \Delta \ln |x-z| \\
& =\int_{\mathbb{R}^{2}}(2 \pi \delta(x-y)-2 a) \rho(y, t) d y+2 \pi b \delta(x-z) \\
& =2 \pi \rho(x)-2 a, \quad x \neq z
\end{aligned}
$$

For a steady state, the velocity is zero so that the swarm density $\rho(x)=a / \pi$ must be constant. Furthermore, any initial conditions evolve to a constant-density state as Chen and Kolokolnikov showed in [1] (see also [24] and [25]). For the reader's convenience, let us repeat the argument here.

Define the characteristic curves $X\left(X_{0}, t\right)$ which start from $X_{0}$ at $t=0$ :

$$
\frac{d X}{d t}=v(X, t) ; \quad X\left(X_{0}, 0\right)=X_{0} .
$$

Then along the characteristic curves $x=X\left(X_{0}, t\right), \rho(x, t)$ satisfies:

$$
\frac{d \rho}{d t}=-\left(\nabla_{x} \cdot v\right) \rho
$$

so that from (3) we obtain

$$
\frac{d \rho}{d t}=(2 a-2 \pi \rho) \rho
$$

It therefore follows that $\rho \rightarrow a / \pi$ as $t \rightarrow \infty$ along the characteristics, provided that initially $\rho>0$ at $t=0$.

Further, it follows that after a transient period we may replace $\rho$ in $(2 \mathrm{~b})$ by a constant $a / \pi$ inside the swarm, and zero outside of the swarm. Equation $(2 \mathrm{~b})$ then becomes

$$
v(x, t)=\frac{a}{\pi} \int_{D}\left(\frac{x-y}{|x-y|^{2}}-a(x-y)\right) d y+b \frac{x-z}{|x-z|^{2}}
$$

where $D$ is the extent of the swarm. This is the starting point of this paper. We also utilize this formula to perform numerical simulations of the continuum model by tracking the boundary of the swarm. In this paper we use this continuum formulation and look more closely at the dynamics Chen and Kolokolnikov [1] discussed for the predator prey particle model formed using equations (1a) and (1b). We also use the boundary model to consider the case of a single predator travelling on an infinite plane of evenly distributed prey.

We remark that more general interaction terms can be considered; for example one may replace $\frac{x_{j}-x_{k}}{\left|x_{j}-x_{k}\right|^{2}}-a\left(x_{j}-x_{k}\right)$ in (1) by a more general pairwise repulsive-attractive force interaction $F\left(\left|x_{j}-x_{k}\right|\right) \frac{x_{j}-x_{k}}{\left|x_{j}-x_{k}\right|}$, and similarly for other terms; see [1]. The continuum limit anologue (2) can be derived for more general nonlinearities; however, the mathematical analysis in [1] and below assumes constant density inside the swarm, which requires the particular choice $F(r)=1 / r-a r$.

Note that the linear attraction $-a\left(x_{j}-x_{k}\right)$ in (1) is biologically unrealistic for large distances since it grows with distance. This can be easily addressed by replacing $-a\left(x_{j}-x_{k}\right)$ by $-a\left(x_{j}-x_{k}\right) f\left(\left|x_{j}-x_{k}\right|\right)$ where $f(r)$ is constant 
near the origin and decays after some cut-off, for instance $f(r)=\left\{\begin{array}{c}1, \quad \text { if } r \leq r_{0} \\ \exp \left(-\left(r-r_{0}\right)\right) \text { if } r>r_{0} .\end{array}\right.$. In particular if $r_{0}$ exceeds the swarm size, then this is equivalent to linear attraction. Similar comments apply to other terms in (1).

\section{Boundary evolution method}

The particle model (1), or its continuum limit (2), typically produces two relatively well defined boundaries (see Figure 1): one between the predator and the prey, the other enclosing the swarm. To compute these boundaries in the continuum limit (2) we perform a numerical tracking of the boundaries in time by computing the velocity along the boundary using (7). By making use of the divergence theorem, we can rewrite the two-dimensional integral in (7) as an integral along the boundary itself,

$$
v(x)=\frac{a}{\pi} \int_{\partial D}\left(-\ln |x-y|+\frac{a}{2}|x-y|^{2}\right) \hat{n} d S(y)+b \frac{x-z}{|x-z|^{2}}
$$

Similarly, substituting $\rho=a / \pi$ into (2c) and using the divergence theorem yields

$$
v(z)=\frac{c a}{\pi} \int_{\partial D} \frac{|y-z|^{2-p}}{2-p} \hat{n} d S(y) .
$$

By discretizing the inner and outer boundaries, we approximate the integral in (8a) numerically for each point on the boundary. The boundary $D$ and the predator location $z$ then evolve according to the velocities thus computed.

We first used the standard Forward Euler method to update to points on the boundary. Numerically we found that this computation was often unstable regardless of the choice of time step $\Delta t$. This instability arises because the points along the boundary tend to cluster together as time evolves. The clustering of points resulted from the dynamics pushing particles back along the boundary and forcing them to aggregate at the tail. As a remedy we introduced reparameterization based on arc length at every time step.

To reparameterize a curve based on arc length, we introduced the arclength monitor function, $M(i)$ such that $M(0)=0$ and $M(i+1)=M(i)+\left|x_{i}-x_{i-1}\right|$. We then reparametrize the curve such that $M$ is equally-distributed. The following self-contained Matlab code fragment illustrates the details:

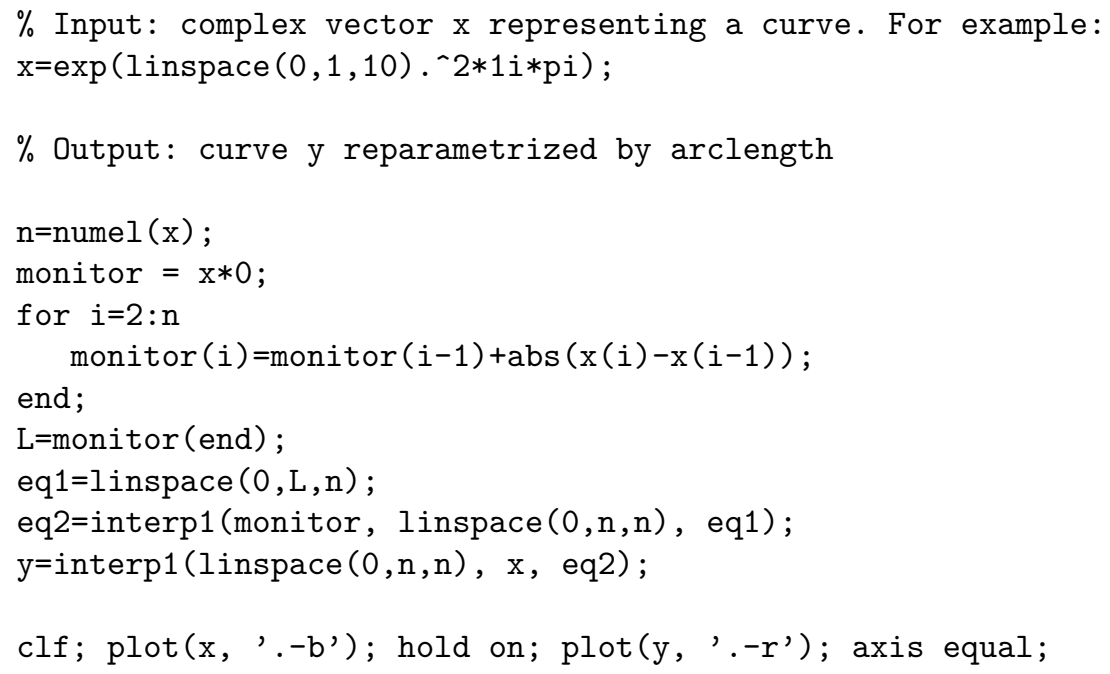

This reparametrization stabilized the numerics. Figure 1 shows a good agreement between the particle model and the boundary evolution method. An important difference here is the tail. In the particle model, the tail merges with the boundary creating an "island" in the middle. On the other hand, in the boundary model the tail curls inwards and fails to merge. The tail itself that forms extends and shortens with time, similar to the case we see in Figure 7 of Section 3.2. A possible future work is to extend the boundary model to allow the merging of the boundaries and formation of "islands". 


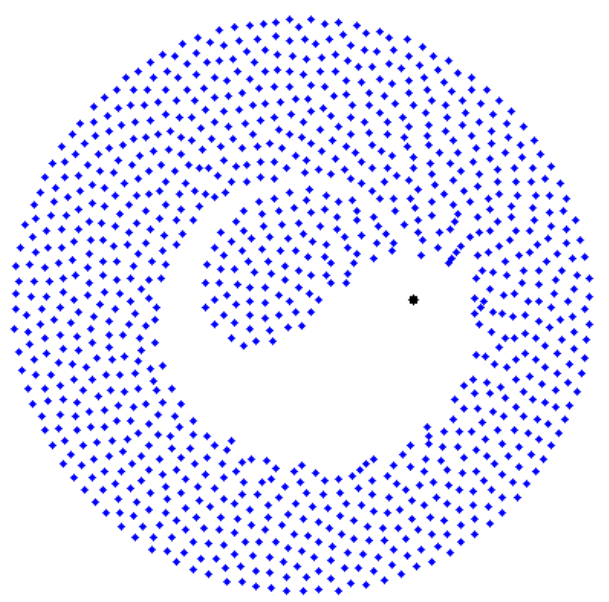

(a)

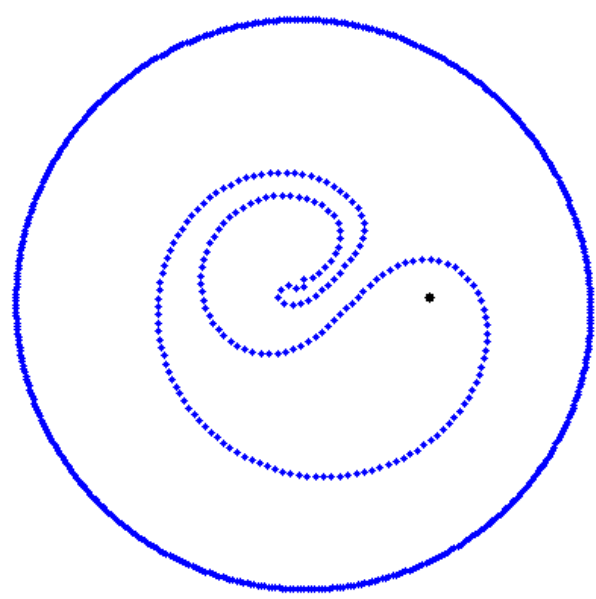

(b)

Figure 1: Comparison between frames taken from two different models, (1) and (8), pertaining to a single predator inside a swarm of prey. (a) Frame of the full particle model (1) that [1] originally proposed. (b) The boundary model computed in the continuum limit (2) using the derivation (8). Parameters for both (a) and (b) are $c=1.5, a=1, b=$ 0.2. A total of 1000 particles was used in (a). In (b), we used 200 points for the inner boundary and 500 for the outer boundary.

\section{Infinite swarm limit}

In [1], Chen and Kolokolnikov showed that the model (2) admits a steady state for which the swarm boundary consists of an annulus with inner radius $R_{1}$ and outer radius $R_{2}$ given by

$$
R_{1}=\sqrt{\frac{b}{a}} \quad R_{2}=\sqrt{\frac{1+b}{a}}
$$

and with the predator located at the center of this boundary. In the double limit $a \rightarrow 0$ and $b=\mathcal{O}(a)$, note that $R_{2} \rightarrow \infty$ while $R_{1}$ remains $\mathcal{O}(1)$. Thus the exterior boundary becomes infinite while the interior boundary remains finite in size.

Taking the limit $a \rightarrow 0$ in (8a) and discarding the $\mathcal{O}\left(a^{2}\right)$ terms as well as the exterior boundary, we obtain

$$
v(x)=\frac{1}{\pi} \int_{-\partial D_{i}}-a \ln |x-y| \hat{n} d S(y)+b \frac{x-z}{|x-z|^{2}}
$$

where $-\partial D_{i}$ denotes an interior boundary, traversed clockwise. By rescaling space we may take without loss of generality $a=b$. By rescaling time $t=a \hat{t}$ and dropping the hat, the equation for velocity then becomes

$$
v(x)=\frac{1}{\pi} \int_{\partial D_{i}} \ln |x-y| \hat{n} d S(y)+\frac{x-z}{|x-z|^{2}} .
$$

The swarm has an interior boundary $\partial D_{i}$ while the exterior boundary we assume to be arbitrary large. We refer to (11) as the infinite swarm limit model. It requires a coupling with some law of motion for the predator $z$. The simplest such motion is to assume that $z$ moves at a constant speed along a straight line. We consider this in Section 3.1, and study the more complicated case of a predator moving in a circle in Section 3.2. Even in this simplest settings, one can observe complex boundary shapes for which we do not know any closed-form solutions. Furthermore, when the predator is moving sufficiently fast, we observe a formation of an infinite tail behind the inner swarm boundary.

\subsection{Predator moving in straight line}

To investigate the properties of a single boundary model, first consider the case of $z$ moving in a straight line at a constant speed, $\omega$. For simplicity let $z$ move along the horizontal axis. Thus, the following equation models the movement of $z$

$$
z=\omega t
$$


To aid in the comparison of these graphs between different values of $\omega$ consider looking at the movement of $z$ in a moving frame such that $z$ appears stationary. Again, for convenience set $z$ at the origin by making the following change of coordinates

$$
x=\tilde{x}+\omega t
$$

so that $v(x)=d x / d t=d \tilde{x} / d t+\omega=v(\tilde{x})+\omega$, and $z=0$. Then the infinite swarm model (11) becomes, after dropping the tildes,

$$
v(x)=\frac{1}{\pi} \int_{\partial D_{i}} \ln |x-y| \hat{n} d S(y)+\frac{x}{|x|^{2}}-\omega
$$

Figure 2 shows the evolution of the boundary for $\omega$ equal to $0.2,0.4$, and 0.6 . For small $\omega$, after a transient time, the boundary shape converges to the steady state in the translational frame for which $v$ in (14) is zero for $x$ along $\partial D_{i}$. However as $\omega$ increases, a tail starts to form at the back of the boundary. As Figure 3 illustrates, this tail appears to grow indefinitely as $t$ is increases.

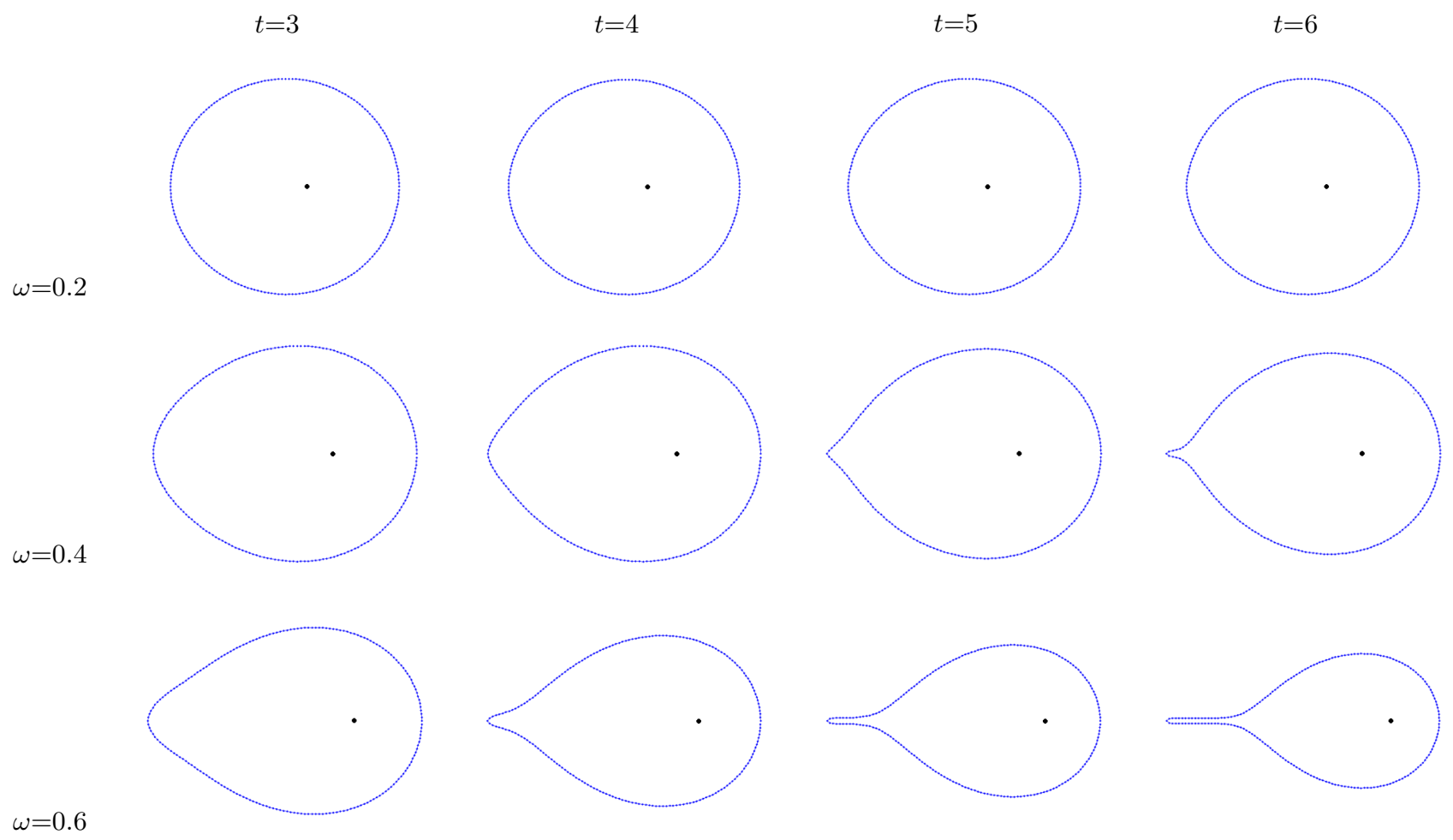

Figure 2: Stationary plots modelling the case of a single predator z moving in a straight line through an infinite swarm of prey. We compare how the boundary forms over time for different speeds, $\omega$. Here we use (14), which uses a change of coordinates to fix $\mathrm{z}$ at the origin, effectively modelling the view of a moving frame around $\mathrm{z}$. Parameters are $t$ and $\omega$ as given. While $\omega=0.2$ reaches a stationary state, $\omega=0.4$ and $\omega=0.6$ continue to grow with time.

The bifurcation appears to occur when $\omega$ increases past some critical value $\omega=\omega_{c}$. Numerical testing showed this value is approximately 0.25. While Figure 3 shows the tail at specific times, it is important to note that this tail does not appear to reach a stationary state, but rather continually lengthens.

The tail itself is reminiscent of the tail seen in the particle model (1a) and (1b) form, as well as the one that we saw form in the case of $z$ moving in a circle in section 3.2 below. It is possible that the rotating nature of these other cases prevent the tail from developing, as otherwise they would force the tail to cross its own boundary. Though no predator-prey models were found that indicated an infinite tail formation, we note the solution found in a swarm model by Mogilner and Edelstein-Keshet [26], which indicated situations in which an infinite tail of prey would form.

Further numerical simulations show that there is a saddle point, as Figure 4 shows. The saddle point lies on $\mathrm{x}$-axis to the left of the boundary for values of $\omega$ lower than $\omega_{c}$. As the value of $\omega$ increases towards $\omega_{c}$, this saddle point crosses the boundary, inducing a topological change in the shape of the swarm. 


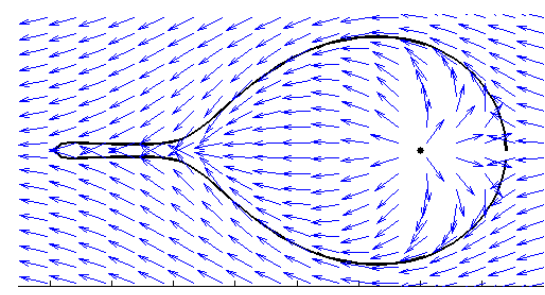

(a)

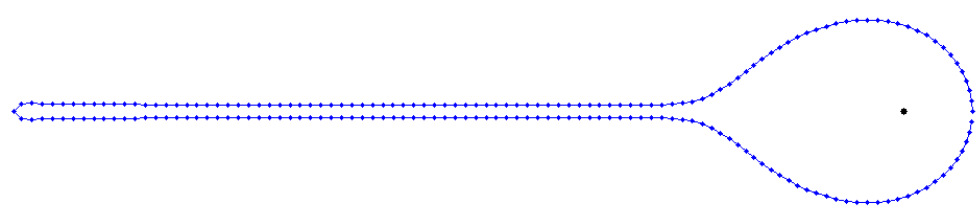

(b)

Figure 3: A tail forming as time increases in the model of a single predator moving in a straight line at constant velocity through an infinite swarm of prey, as described by (14). For large enough values of $\omega$ tested, such as depicted here for $\omega=0.5$, the tail lengthens as time increases. (a) Depicts the vector field around the boundary after the tail has formed and begins to lengthen. Parameters are $n=100, t=8.0$, and $\omega=0.5$. (b) The boundary model at $t=24.0$, emphasizing the development of a long tail. Parameters are $n=200, t=24.0$, and $\omega=0.5$.

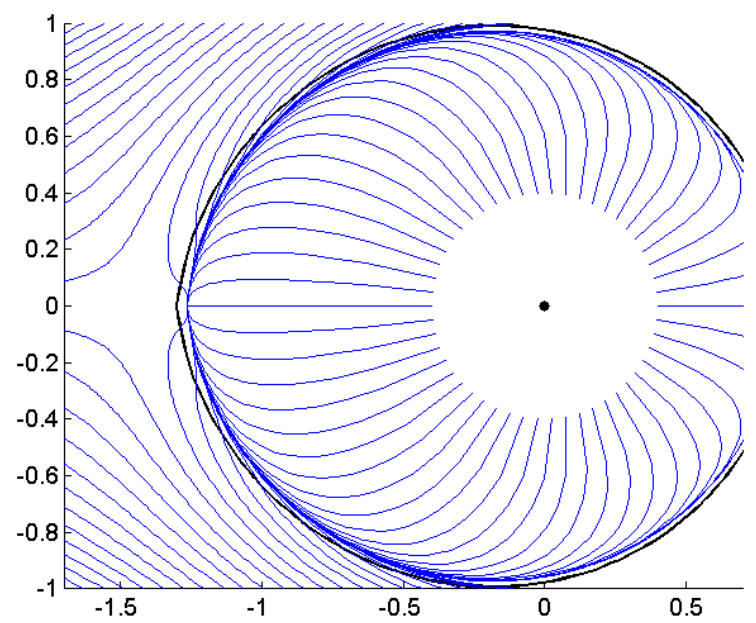

(a)

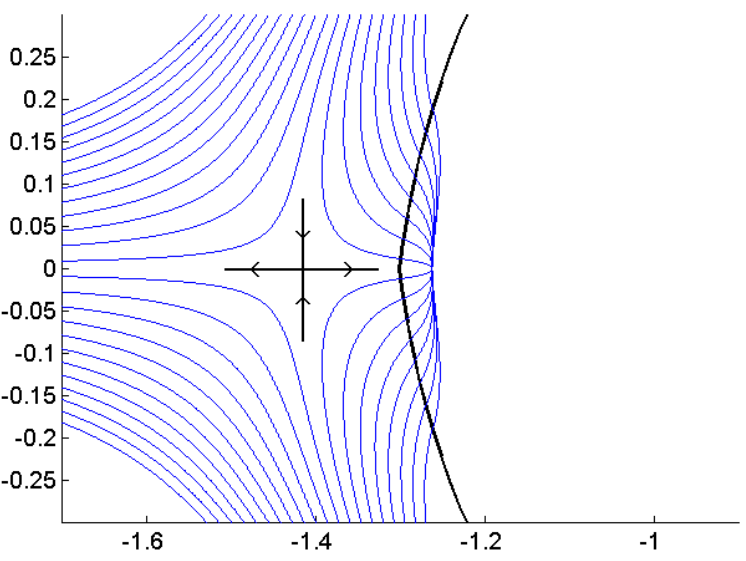

(b)

Figure 4: Orbit plots highlighting the dynamics in the boundary model of a single predator moving linearly at a constant speed, using (14). The parameters are $n=200$ and $\omega=0.2$. The location of the saddle point and end that associate with this $\omega$ are -1.4158 and -1.3019 respectively. The value of $\omega$ pertains to the case of where the model reaches stationary state. (a) Orbits around the whole boundary model indicate the presence of a saddle point and show the dynamics produced. Inside the boundary, the prey move towards the the boundary away from the predator. Outside, a prey may move towards the boundary or away from the swarm initially, depending on its starting position and closeness of the swarm. (b) A closer view of orbits around the saddle point in this system, providing the specific direction the orbits travel. The model shows orbits extending slightly past the end and inside the boundary, which is likely only a numerical artefact of this model. 


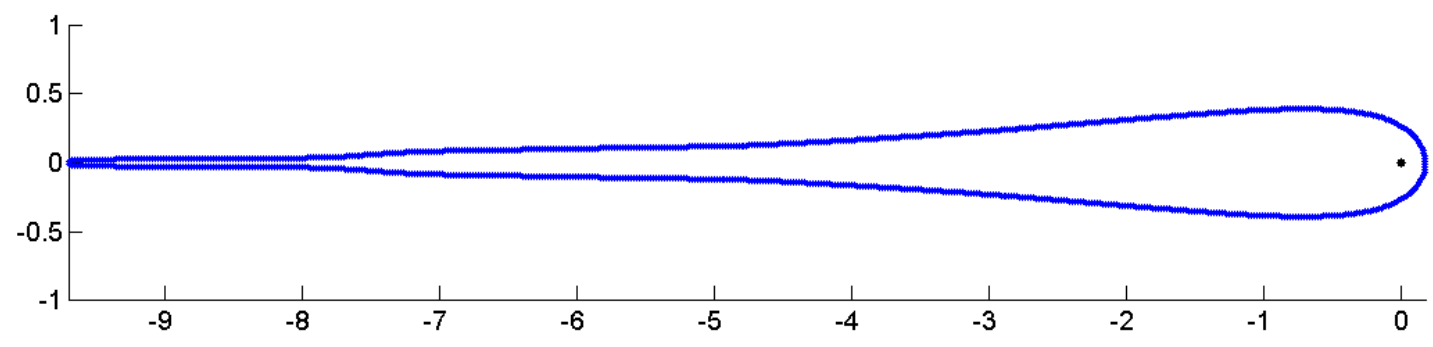

$\omega=10$

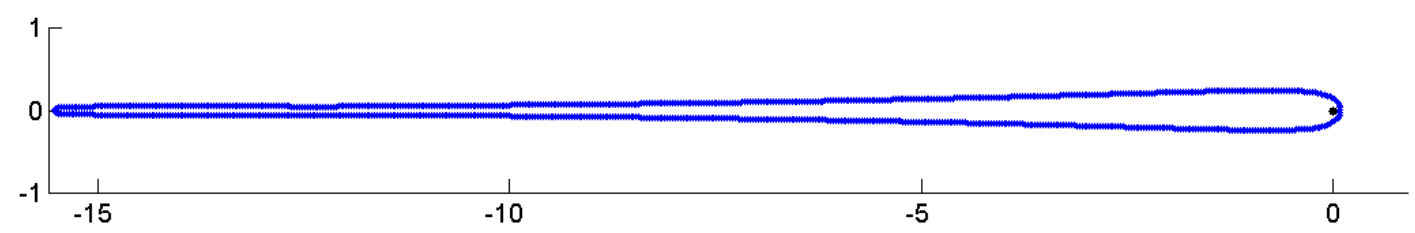

Figure 5: Frames showing the predator reaching larger values of $\omega$ in the case of a predator moving in a straight line through an infinite swarm of prey, using (14). In both these cases the predator is unable to catch the prey, but reaches a close range. Parameters for both are $t=1.5$ and $n=1000$, and $\omega$ is as given. Here, the distance between the predator and the closest prey is 0.1737 and 0.0903 for $\omega=5$ and $\omega=10$ respectively.

There is a secondary bifurcation that appears as $\omega$ increases further, well beyond $\omega_{c} \approx 0.25$. We see the dynamics pertaining to large values of $\omega$ in Figure 5. For increasingly large $\omega$ the predator location starts to approach the right boundary of the swarm. As we further increase the value of $\omega$ the distance between the predator and the prey decreases, as Figures 2 and 5 illustrate. At a certain point, $\omega$ becomes large enough that the predator is able to catch the prey. To quantify this value of $\omega$, which we will denote as $\omega_{\text {catch }}$, we will consider it to be the smallest value of $\omega$ for which the the distance between the predator and the closest prey falls below 0.05 . In finding $\omega_{\text {catch }}$ we must consider two factors: $n$, the number of points with which we model the boundary, and $d t$, our timestep. While $n$ is able to be changed without greatly varying the results, $d t$ must be sufficiently small to maintain certain dynamics, such the predator remaining inside the boundary. Chiefly, $d t$ produces stable results when it is $\leq 0.001$. With $d t=0.001$ and $n=200$ the value of $\omega_{\text {catch }}$ is approximately 19.2. Beyond these values of $\omega$ and $d t$ the numerics start to become unstable and quickly break down, often resulting in the predator escaping without coming in contact with the boundary. In other instances of the numerics breaking down, specifically when $d t$ is large, boundary growth is no longer smooth but occurs in jumps.

\subsection{Predator moving in a circle}

We now consider a predator moving in a circle within the infinite plane of prey. Again, it is possible to use (11) as a model for the velocity of the prey, while we determine the movement of $z$ using the following

$$
z=R e^{i \omega t}
$$

Here, $R$ is the radius of the circle travelled by $z$ and $\omega$ is the angular velocity of $z$. Just as in the case of $z$ moving in a straight line, we change to a moving frame. Consider a change to a rotating frame where $z$ is stationary at $z=R$. Again, we modelled this using a change of coordinates, as follows

$$
x=\tilde{x} e^{i \omega t}
$$

This change of coordinates leads us to the following model

$$
v(x)=\frac{1}{\pi} \int_{\partial D_{i}} \ln |x-y| \hat{n} d S(y)+\frac{x-R}{|x-R|^{2}}-i \omega x .
$$




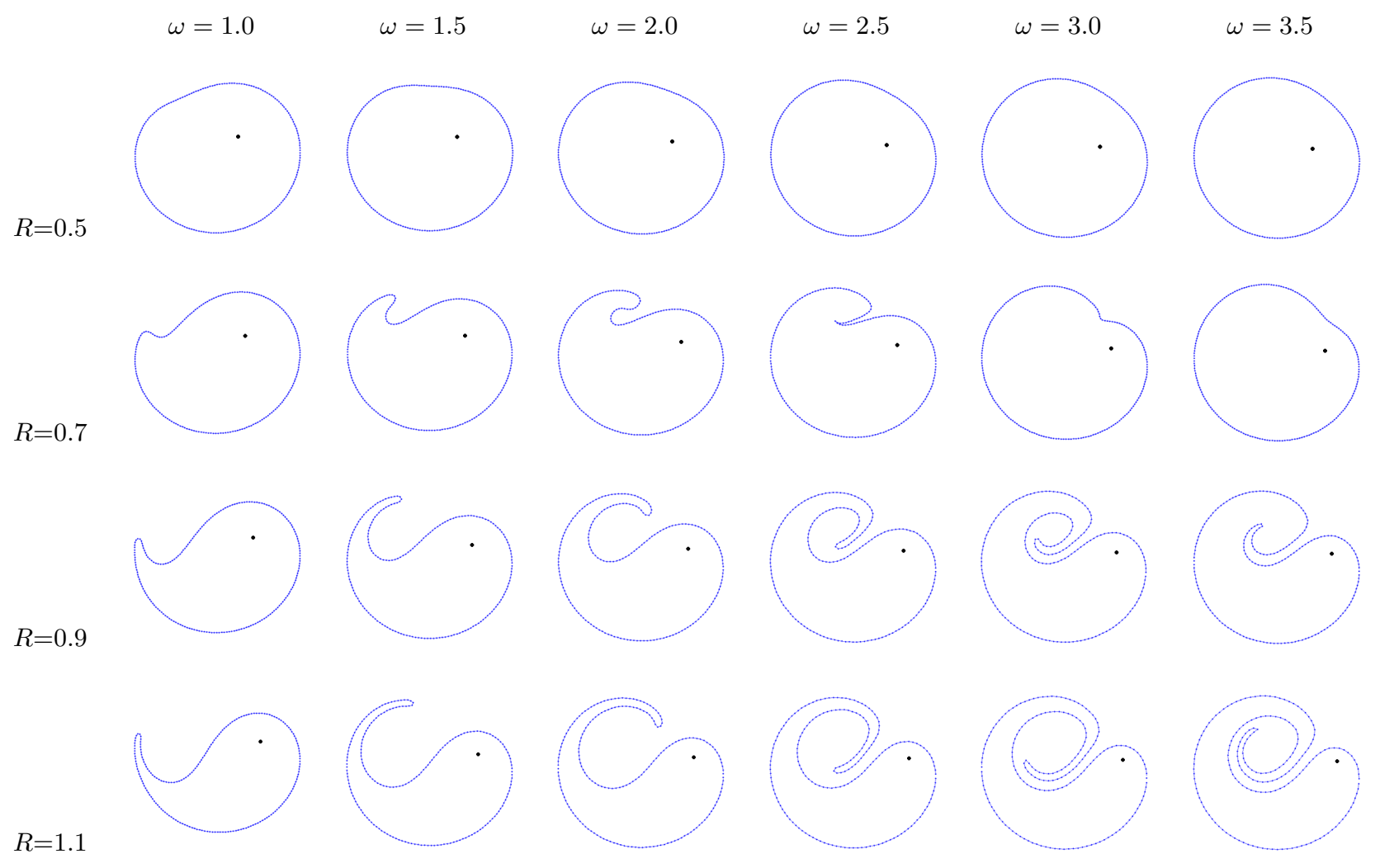

Figure 6: Comparisons of the different boundaries that form in the case of a single predator moving circularly through an infinite swarm of prey, using (17), for different speeds $\omega$ and radii $R$ of the predator. Here, we ran models to a stationary state and fixed $x_{1}$ to remain at the rightmost point. For each $R$ we used the previous stationary state as the initial set-up for the proceeding value of $\omega$. Each model used $n=200$ boundary points and parameters $\omega$ and $R$ are as given.

$t=54.2$

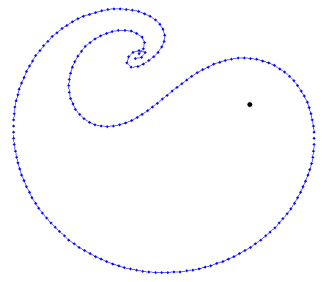

$t=55.7$

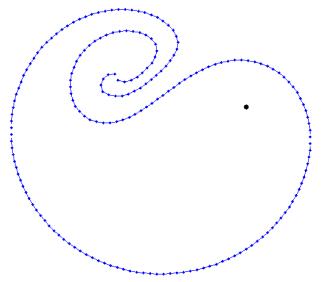

$t=57.2$

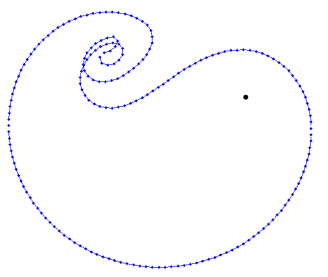

$t=58.7$

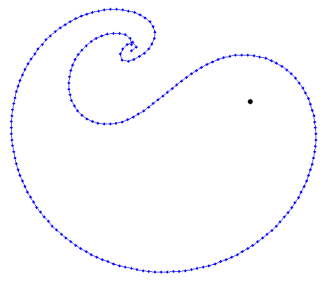

Figure 7: An example in which a continually developing tail forms in the boundary model of a predator moving in a circle through an infinite swarm of prey, which we model using (17). Parameters are $n=200, \omega=2.0, R=0.9$, and time as given. The nature of the tail development was near cyclic; here we show frames depicting one growth cycle. 
Figure 6 shows the variety of the steady states (in the rotational frame) that can occur for various values of $R$ and $\omega$. When $R$ is small, such as $R=0.5$, the steady state seems to be influenced very little by a changing $\omega$, and maintains a relatively circular boundary. Larger values of $R$, such as $R=0.9$, show great dissimilarities between the boundaries different values of $\omega$ form. In many sets of parameters a tail develops without ever stabilizing, continually decreasing and increasing in length. An example of this is visible in Figure 7.

In order to achieve a more stable figure for the different combinations of $R$ and $\omega$, the first point on the boundary, $x_{1}$, is fixed to stay at the rightmost point in the diagram after each reparametrization. With this modification, most parameters allow the boundary to be unchanging in shape over time. As well, this allows us to more easily visualize the effects of different values of $R$ and $\omega$ in model (17). Whereas low values of $R$ and $\omega$ produce a relatively circular boundary, the model begins to grow a tail and to increase in complexity as we increase $R$ and $\omega$. In particular, having $R=0.7$ and $\omega=2.5$ causes the boundary to slide back and forth, so that a stable rotating state is never reached. In other cases, the tail continues to grow and wraps around the itself again, forming a second boundary. We show an example of a second boundary in Figure 8. With lower values of $\omega$ the formation of a second boundary is often preventable by slowly increasing $\omega$ towards its desired value.

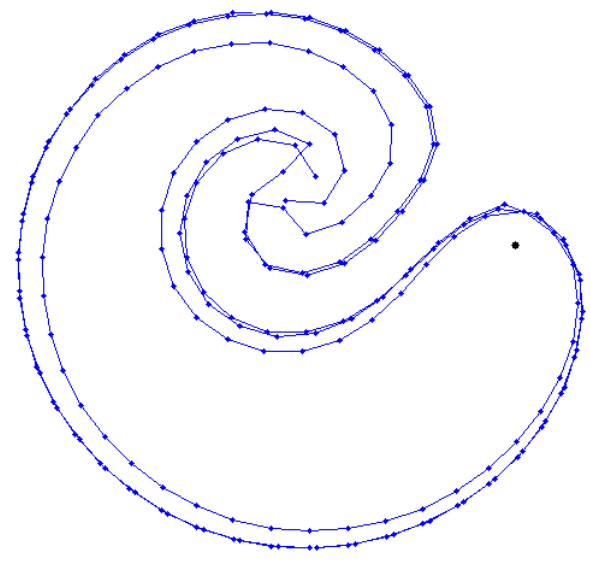

Figure 8: We show the peculiar double boundary observed when modelling the case of a predator moving in a circle through an infinite swarm of prey, which (17) models. Parameters are $n=200, R=0.7, \omega=6.0$. The still shown is at $t=50$ with $\omega$ equal to the minimum of $2+0.1 * t$ and 6.0 .

To more closely examine what is happening in the cases of (17) where the dynamics become complex, we examine the vector fields around the boundary plots. In Figure 9 we show the vector fields around boundary plots with $\omega$ increasing linearly with time. In this figure, we see a distinct "whirlpool" or spiral in the vector field that appears to force the tail to curl around. To the right of this, along the boundary, dynamics suggest a saddle point.

The saddle point and spiral are clearly visible in the larger frames of vector plots Figure 10 depicts. Here we use the steady state with $\omega=3.5$. In the case we present in Figure 10 the point sits inside the boundary. In the former case of a moving tail we showed, the saddle point appears along the boundary. The saddle point in this instance causes points to move towards it vertically and points to move away from it horizontally along the boundary.

In the case of where $x_{1}$ is not fixed an evident difference in the boundary model (17) is that the gap in the boundary between $x_{1}$ and $x_{n}$ is able to move to the end of the tail. In the case of a fluctuating tail, this seemingly allows the tail to grow long and causes it to run into its own boundary. This expels the tail back outside, and the cycle repeats itself. The vector field itself seems to evolve little.

\section{Discussion and extensions}

In this paper we investigated the dynamics and the steady states of the predator-swarm model (1) introduced in [1]. The advantage of this model is that in the large $N$ limit, the swarm density is constant, which makes it possible to describe the dynamics purely in terms of the evolution of the boundary of the swarm. We implemented a numerical method to track the boundary, and investigated the resulting steady states in rotating frame. We then considered a 

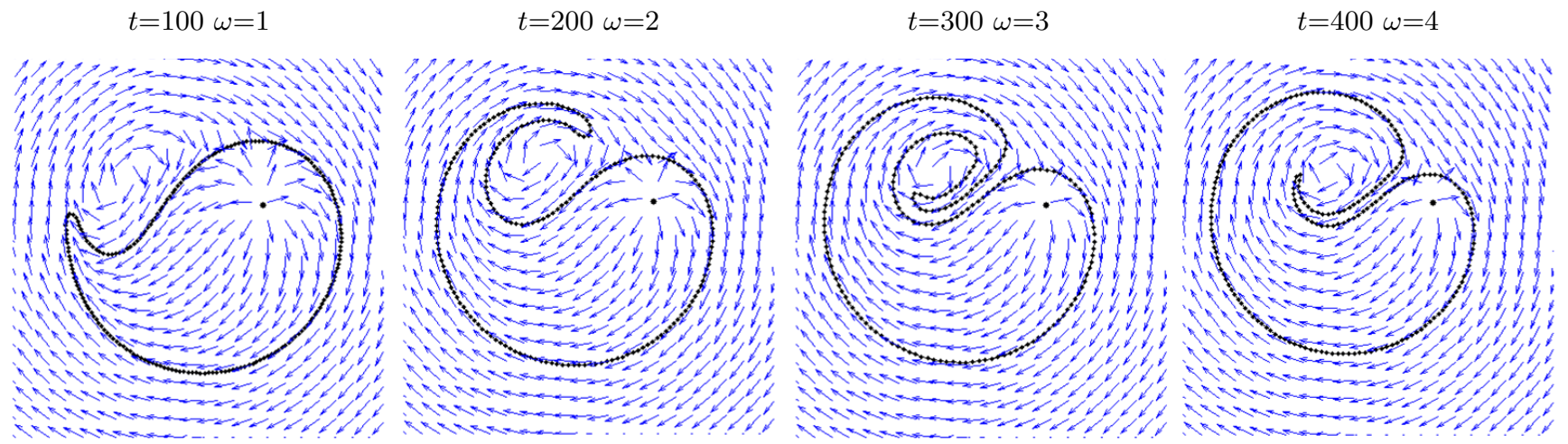

Figure 9: Vector field plots are used to show system dynamics of (17), which models the case of a predator moving in a circle through an infinite swarm of prey. Here we increase $\omega$ linearly with time, with $\omega=0.01 t, R=0.9$, and $x_{1}$ fixed to remain at the rightmost point. Parameters were $n=200$ and $t$ as given. We note the spiral visible near the tail as well as the appearance of a saddle point on the boundary, directly above the predator.

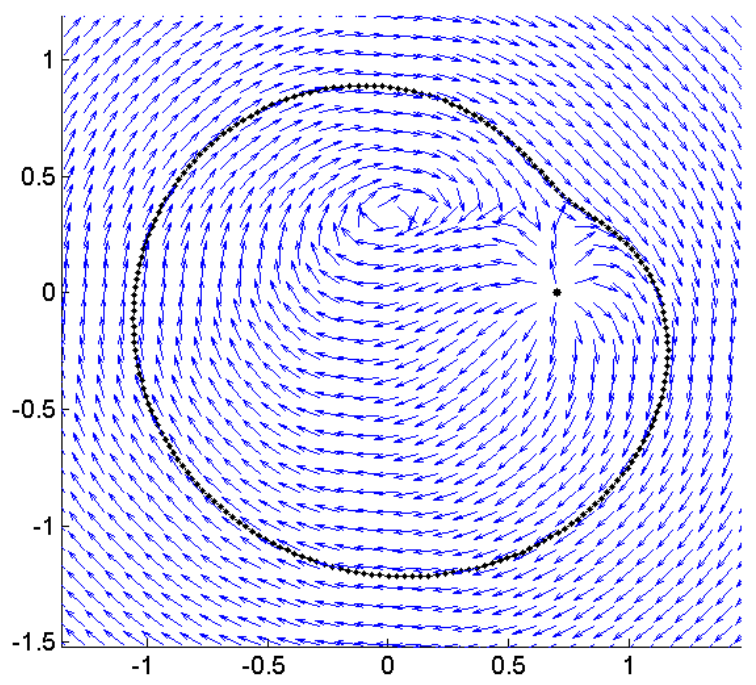

(a)

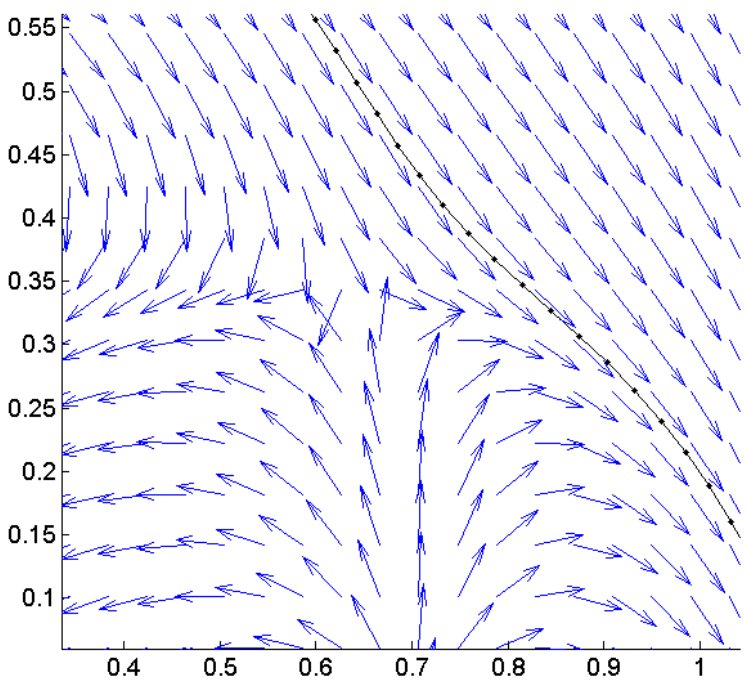

(b)

Figure 10: Vector field plot of the steady state for (17) with $\omega=3.5, R=0.7$ and $n=200$. Just as Figure 9 showed in the case of a tail forming, a saddle point is visible in this stationary state. In (a) we see the full image of the vector plot. As well, we note the visibile spiral dynamics as before. One can more easily see the saddle point in (b), which shows a close up of the vector dynamics in this system. 
limiting case of a predator moving inside a sea of infinite swarm. In this case, only the interior boundary needs to be tracked. We considered two cases: a predator moving along a straight line, and a predator moving in a circle. The former is particularly simple since there is only one effective parameter, which is the speed of the predator. Even in this simplified setting, we showed that the interior boundary forms an infinite tail as the speed of the predator increases beyond a certain threshold. In the case of a predator moving in a circle, the steady state exhibits a very rich structure with multiple transitions depending on the radius and angular velocity of the predator.

Our numerical investigations pose many open questions. For example, in the case of a predator moving in a straight line, is it possible to find a closed-form solution for the shape of the steady state? We demonstrated numerically that the appearance of an infinite tail relates to topological changes as a saddle point crosses the swarm boundary. Can this be studied analytically? These and related questions pose challenging problems for future study.

\section{Acknowledgements}

We would like to thank the referees for their careful reading of our work and their valuable suggestions and comments that led to great improvements from the original.

\section{References}

[1] Y. Chen, T. Kolokolnikov, A minimal model of predator-swarm interactions, Journal of The Royal Society Interface 11 (94) (2014) 20131208.

[2] Y. Katz, K. Tunstrøm, C. C. Ioannou, C. Huepe, I. D. Couzin, Inferring the structure and dynamics of interactions in schooling fish, Proceedings of the National Academy of Sciences 108 (46) (2011) 18720-18725.

[3] U. Lopez, J. Gautrais, I. D. Couzin, G. Theraulaz, From behavioural analyses to models of collective motion in fish schools, Interface Focus (2012) rsfs20120033.

[4] A. Okubo, D. Grünbaum, L. Edelstein-Keshet, The dynamics of animal grouping, in: Diffusion and ecological problems: modern perspectives, Springer, 2001, pp. 197-237.

[5] C. W. Reynolds, Flocks, herds and schools: A distributed behavioral model, ACM SIGGRAPH Computer Graphics 21 (4) (1987) 25-34.

[6] C. Huepe, G. Zschaler, A.-L. Do, T. Gross, Adaptive-network models of swarm dynamics, New Journal of Physics 13 (7) (2011) 073022.

[7] J. Buhl, G. A. Sword, S. J. Simpson, Using field data to test locust migratory band collective movement models, Interface focus (2012) rsfs20120024.

[8] L. Edelstein-Keshet, Mathematical models of swarming and social aggregation, in: Proceedings of the 2001 International Symposium on Nonlinear Theory and Its Applications, Miyagi, Japan, 2001, pp. 1-7.

[9] A. J. Bernoff, C. M. Topaz, A primer of swarm equilibria, SIAM Journal on Applied Dynamical Systems 10 (1) (2011) 212-250.

[10] V. Zhdankin, J. Sprott, Simple predator-prey swarming model, Physical Review E 82 (5) (2010) 056209.

[11] G. Flierl, D. Grünbaum, S. Levins, D. Olson, From individuals to aggregations: the interplay between behavior and physics, Journal of Theoretical Biology 196 (4) (1999) 397-454.

[12] R. Lukeman, Y.-X. Li, L. Edelstein-Keshet, Inferring individual rules from collective behavior, Proceedings of the National Academy of Sciences 107 (28) (2010) 12576-12580.

[13] P. Kareiva, G. Odell, Swarms of predators exhibit "preytaxis" if individual predators use area-restricted search, The American Naturalist 130 (2) (1987) pp. 233-270.

[14] A. Ordemann, G. Balazsi, F. Moss, Pattern formation and stochastic motion of the zooplankton daphnia in a light field, Physica A: Statistical Mechanics and its Applications 325 (12) (2003) 260 - 266, stochastic Systems: From Randomness to Complexity. 
[15] A. Eriksson, M. N. Jacobi, J. Nyström, K. Tunstrøm, Determining interaction rules in animal swarms, Behavioral Ecology 21 (5) (2010) 1106-1111.

[16] S. Nishimura, T. Ikegami, Emergence of collective strategies in a prey-predator game model, Artificial Life 3 (4) (1997) 243-260.

[17] M. Higashitani, A. Ishigame, K. Yasuda, Particle swarm optimization considering the concept of predator-prey behavior, in: Evolutionary Computation, 2006. CEC 2006. IEEE Congress on, 2006, pp. 434-437.

[18] M. M. Molina, M. A. Moreno-Armendriz, J. C. S. T. Mora, On the spatial dynamics and oscillatory behavior of a predator-prey model based on cellular automata and local particle swarm optimization, Journal of Theoretical Biology 336 (0) (2013) $173-184$.

[19] A. Silva, A. Neves, E. Costa, An empirical comparison of particle swarm and predator prey optimisation, in: Artificial Intelligence and Cognitive Science, Springer, 2002, pp. 103-110.

[20] A. Deutsch, G. Theraulaz, T. Vicsek, Collective motion in biological systems, Interface Focus 2 (6) (2012) 689-692.

[21] J. K. Parrish, L. Edelstein-Keshet, Complexity, pattern, and evolutionary trade-offs in animal aggregation, Science 284 (5411) (1999) 99-101.

[22] D. J. Sumpter, R. P. Mann, A. Perna, The modelling cycle for collective animal behaviour, Interface focus (2012) rsfs20120031.

[23] A. Rozenfeld, E. Albano, Study of a lattice-gas model for a preypredator system, Physica A: Statistical Mechanics and its Applications 266 (14) (1999) 322 - 329.

[24] R. C. Fetecau, Y. Huang, T. Kolokolnikov, Swarm dynamics and equilibria for a nonlocal aggregation model, Nonlinearity 24 (10) (2011) 2681.

[25] A. L. Bertozzi, T. Laurent, F. Léger, Aggregation and spreading via the newtonian potential: the dynamics of patch solutions, Mathematical Models and Methods in Applied Sciences 22 (supp01).

[26] A. Mogilner, L. Edelstein-Keshet, A non-local model for a swarm, Journal of Mathematical Biology 38 (6) (1999) $534-570$. 